\title{
Design Optimization Analysis of Venturi Tube for Medium Conveying in Strengthen Grinding Process
}

\author{
Jinrui Xiao ${ }^{1,2 *}$, Zhongwei Liang1,2, Xiaochu Liu ${ }^{1,2}$, Zhuan Zhao², Xincheng Xie ${ }^{2,3}$ \\ ${ }^{1}$ School of Mechanical and Electrical Engineering, Guangzhou University, Guangzhou, China \\ ${ }^{2}$ Guangzhou Key Laboratory of High-Performance Metal Grinding Processing, Guangzhou, China \\ ${ }^{3}$ School of Electromechanical Engineering, Guangdong University of Technology, Guangzhou, China \\ Email: *meexiaojinrui@gzhu.edu.cn
}

How to cite this paper: Xiao, J.R., Liang, Z.W., Liu, X.C., Zhao, Z. and Xie, X.C. (2021) Design Optimization Analysis of Venturi Tube for Medium Conveying in Strengthen Grinding Process. Engineering, 13, 431-447. https://doi.org/10.4236/eng.2021.138031

Received: July 12, 2021

Accepted: August 16, 2021

Published: August 19, 2021

Copyright ( 2021 by author(s) and Scientific Research Publishing Inc. This work is licensed under the Creative Commons Attribution International License (CC BY 4.0).

http://creativecommons.org/licenses/by/4.0/ (c) (i) Open Access

\begin{abstract}
Venturi tubes are widely used in manufacture and chemical industry and attract broad attention. To improve the transmission efficiency, the optimization of Venturi tube has been carried out, the transport substances involved in either liquid, solid, gas or two of them. The Venturi tubes that used for conveying of three-phase flow, which is consisted of solid, liquid and gas, are poorly investigated. In this paper, a Venturi tube employed in one surface treatment equipment was proposed. The velocity and pressure distribution in the flow field of Venturi tube with different key geometric parameters including diameter ratio, convergence angle and diffuser angle have been studied. One optimal solution has been selected. The negative pressure of suction port can reach a value as high as $-1550 \mathrm{~Pa}$ to $-1600 \mathrm{~Pa}$. Such negative pressure offers the opportunities for transporting mixed abrasive.
\end{abstract}

\section{Keywords}

Venturi Tube, Strengthen Grinding, Medium Conveying, Parameter Optimization

\section{Introduction}

Over the past decades, Venturi tube has grown of importance in many applications such as cavitation bubbles [1], liquid flow metering [2], powder and particle transportation [3]. It's worth pointing out that Venturi tube plays an extremely important role in the transportation of medium (i.e. powder, particles and liquid) in manufacturing industry, such as shot peening, water jet, sand blasting [4] [5] [6] [7] [8]. Strengthen grinding technology [9] [10], which employs a three-phase 
jet flow that consists of abrasive powders, steel balls and grinding fluids to shoot workpiece, emerges as a new solution to improve the fatigue life of metals (See Figure 1). Such technology can reduce surface roughness and form a harden layer, which is believed to enhance the fatigue life of the metals since the micro-cutting effect and shock processing of abrasive powders and steel balls. Moreover, the grinding fluid helps clear dust and rust. Therefore, strengthen grinding technology opened a new window for processing metal parts such as bearing, gear, and mould.

Venturi tubes are among the most important components in strengthen grinding equipment, but they usually suffered from poor conveying and non-continuity feeding of mixed abrasive during working condition. The conveying efficiency is mainly impacted by the flow resistance. The flow resistance, which may weaken the conveying efficiency of the Venturi tube, is highly impacted by geometry parameters. It mainly reflects in velocity and pressure distribution of the flow field. To address this issue, many studies have been carried out [11] [12] [13]. An example of Venturi tube has been optimized to evaluate its effect on the conveying properties and the pressure reduction by flowing gas-solid mixtures [14]. Li [15] demonstrates that the outlet tangle and the diameter ratio of Venturi tubes are the two key parameters in controlling the throat pressure and power consumption. However, these works are mainly focused on either solid-gas or fluid transportation, the suction pressure inside is relatively low. Venturi tube used for transporting of three-jet flow has a higher requirement on suction pressure and the investigation is still absent.

In this paper, a Venturi tube that used for transporting mixed abrasive has been proposed and optimized. Finite element method (FEM) was taken into consideration and the pressure and velocity distribution in the flow field have been studied. The optimal parameters including the ratio between throat diameter and inlet diameter $\left(k_{1}\right)$, the ratio between suction diameter and throat diameter $\left(k_{2}\right)$, and the convergent angle $(\alpha)$ as well as the diffuser angle $(\beta)$ were selected. Through further verification, the optimal solution performs well and has great potential to be used in transporting mixed medium.

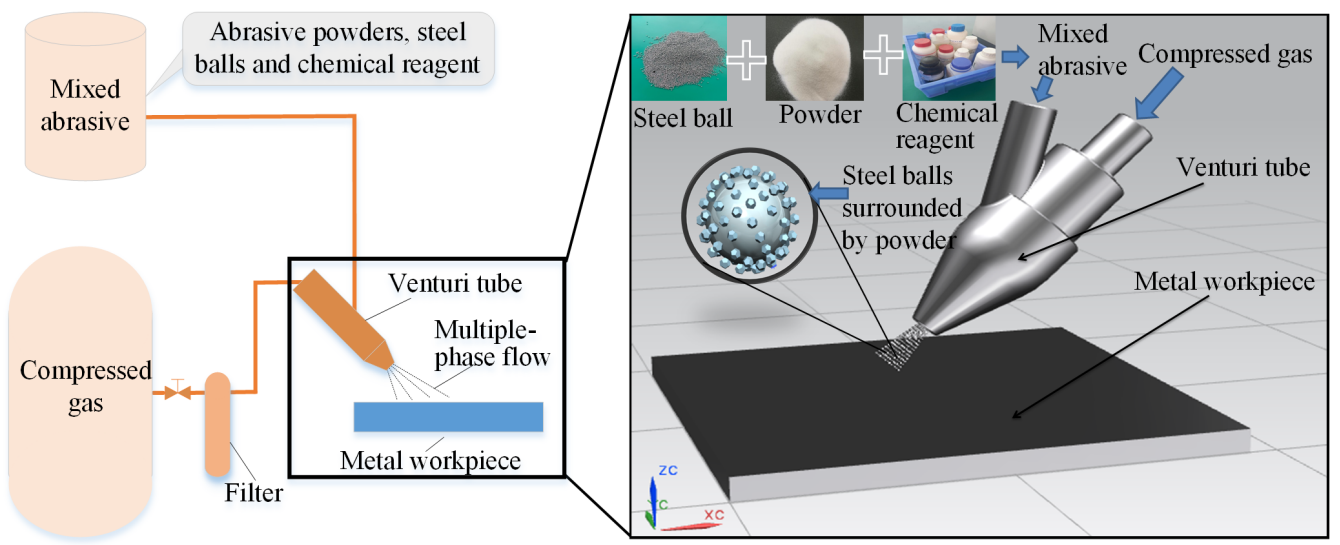

Figure 1. Scheme of the strengthen grinding processing setup. 


\section{Modeling and Principle of the Tube}

The proposed simplified Venturi tube is shown in Figure 2. It includes two parts: main tube and suction tube, the suction tube located in the throat of main tube. Table 1 reported the definitions of the geometric parameters.

When medium flow through main tube, the gas inside is carried away and the static pressure drops as the flow velocity increases. A negative pressure effect is formed once the flow velocity reaches a critical value, which help to absorb material from the suction port. The velocity increases rapidly in the process from inlet to throat since the diameter convergence. The maximum velocity appears in the throat tube and thus causes a maximum negative pressure. Therefore, the solid particles such as steel balls and abrasive powders can be sucked into the main tube by the negative pressure effect. Finally, the transportation of the solid material or mixed abrasive can be realized.

The pipeline pressure versus flow velocity is described by the following Bernoulli equation

$$
P=C-\frac{1}{2} \rho v U^{2}-\rho g h
$$

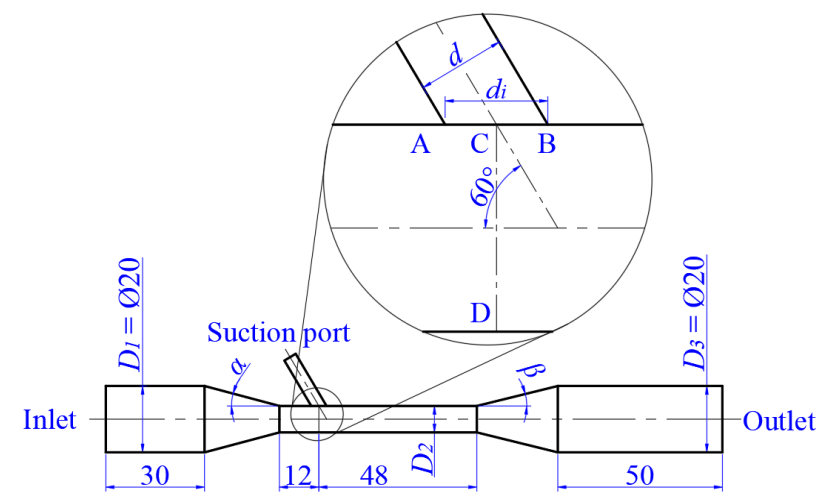

Figure 2. The structure of the proposed Venturi tube.

Table 1. Definition of geometric parameters.

\begin{tabular}{|c|c|}
\hline Parameter & Definition \\
\hline$D_{1}$ & The entrance diameter, which is equal to $20 \mathrm{~mm}$. \\
\hline$D_{2}$ & The throat diameter \\
\hline$D_{3}$ & The outlet diameter, which is equal to $20 \mathrm{~mm}$. \\
\hline$d$ & Diameter of the suction port \\
\hline$d_{i}$ & $\begin{array}{l}\text { The length of the intersection of the suction pipe and the throat } \\
\text { on the symmetry plane. }\end{array}$ \\
\hline$\alpha$ & Convergent angle \\
\hline$\beta$ & Diffuser angle \\
\hline $\mathrm{AB}$ & The intersecting line of suction pipe and throat pipe on the symmetry plane. \\
\hline $\mathrm{CD}$ & A line that is perpendicular to the midpoint of line $A B$. \\
\hline
\end{tabular}


where $P$ is the pressure, $U$ is the flow velocity, $\rho$ is the fluid density, $g$ is the gravitational acceleration, $h$ is the height of the flow and $C$ is a constant.

The suction force of the Venturi tube can be expressed as [16]

$$
F_{d}=\frac{1}{8} \pi C_{d} \rho U^{2} D^{2}
$$

where $C_{d}$ is the drag coefficient of the flow around the medium, $\rho$ is the density of the ambient flow, $D$ is the cross-section diameter.

The drag coefficient of the flow around the medium can be expressed as [17]

$$
C_{d}=\frac{24}{R_{e}}\left(1+b_{1} R_{e}^{b_{2}}\right)+\frac{b_{3} R_{e}}{b_{4}+R_{e}}
$$

where $R_{e}$ is the Reynolds number, $b_{x}$ is the coefficient that depends on the shapes of solid and the location of parts.

The mixed abrasive used for strengthen grinding is a mixture of steel balls and abrasive powder, and its shape is irregular. Here, a variate $\tau$ is introduced to describe the surface area of the mixed abrasive. And it's defined as

$$
\tau=\frac{S_{1}}{S_{2}}
$$

where $S_{1}$ is the surface area of the equivalent sphere, and $S_{2}$ is the surface area of the mixed abrasive. Then, the coefficient of $b_{x}$ can be expressed as [17]

$$
b_{x}= \begin{cases}\exp \left(2.33-6.46 \tau+2.54 \tau^{2}\right) & (x=1) \\ 0.09+0.56 \tau & (x=2) \\ \exp \left(4.91-13.84 \tau+18.42 \tau^{2}-10.26 \tau^{3}\right) & (x=3) \\ \exp \left(1.47+12.26 \tau-20.73 \tau^{2}+15.89 \tau^{3}\right) & (x=4)\end{cases}
$$

The airflow density is related to the pressure and the velocity according to the Bernoulli equation, shown in Equation (6).

$$
\rho=\frac{2 C-2 P}{U^{2}}
$$

Substituting Equation (3) and Equation (6) into Equation (2), the suction force can be expressed as

$$
F_{d}=\left[\frac{3}{R_{e}}\left(1+b_{1} R_{e}^{b_{2}}\right)+\frac{b_{3} R_{e}}{8 b_{4}+8 R_{e}}\right](2 C-2 P) D^{2}
$$

with joint Equation (5) and Equation (7), the suction force of the Venturi tube can be got.

It can be seen that the suction force of Venturi tube is highly depend on the flow velocity, which was impacted by the throat diameter $\left(D_{2}\right)$, the suction port diameter $(d)$, the convergent angle $(\alpha)$, the diffuser angle $(\beta)$ and the inlet velocity. Therefore, the geometry parameters were taken into consideration in this study. To simplify the analysis, $k_{1}$ and $k_{2}$ were introduced, which were defined as $k_{1}=D_{2} / D_{1}$ and $k_{2}=d / D_{2}$, respectively. 


\section{Methods and Simulation}

This work was carried out in the fluent module of ANSYS Workbench software using FEM method. It was regarded as an effective tool for the design and optimization of Venturi tubes [18] [19] [20]. As mentioned before, a three-jet flow needs a higher suction pressure that caused by negative pressure effect, while negative pressure effect is highly depends on flow velocity. Hence, pressure and velocity were simulated. To improve simulation efficiency, a single medium of gas was selected and a 3-D model was considered.

The geometric parameters were selected from Figure 2, the boundary condition is shown in Figure 3. The initial pressure in both the suction port and outlet port was set as zero. The flow velocity in the inlet was set as $20 \mathrm{~m} / \mathrm{s}$. The flow field is in a stable state and work at room temperature. The adaptive method was adopted for the meshing of model (See Figure 3).

The governed equations in the simulation are shown in the following:

$$
\begin{gathered}
\frac{\partial \rho}{\partial t}+\nabla \cdot(\rho U)=0 \\
v=\sum_{1}^{2} \frac{\alpha_{1} \rho_{1} U_{1}}{\rho} \\
\rho=\sum_{1}^{2} \alpha_{1} \rho_{1}
\end{gathered}
$$

where $\alpha_{1}, \rho_{1}$ and $U_{1}$ are the fraction, density and velocity, respectively.

The velocity dependence of pressure can be obtained by discrete momentum equation, which is shown in Equation (11).

$$
\rho=\sum_{1}^{2} \alpha_{1} \rho_{1}
$$

The velocity dependence of pressure can be obtained by discrete momentum equation, which is shown in Equation (11).

$$
a_{P} \overrightarrow{U_{P}^{r}}+\sum_{N} a_{N} \overrightarrow{U_{N}^{r}}=\vec{S}-\nabla P^{0}
$$

where $a_{P}$ and $a_{N}$ are constants, $S$ is a microunit on the surface of the spatial control body, $\overrightarrow{U_{P}^{r}}$ is the predicted center velocity of the control body, $\overrightarrow{U_{N}^{r}}$ is the predicted velocity around the control body and $P^{0}$ is the initial pressure.

The Semi-implicit method for pressure linked equations consistent (SIMPLEC) algorithm, which is based on continuity equation of Equation (12) and Equation (13) [21] [22], was employed for the calculation. And the solving process of velocity and pressure in the Venturi tube was illustrated in Figure 4.

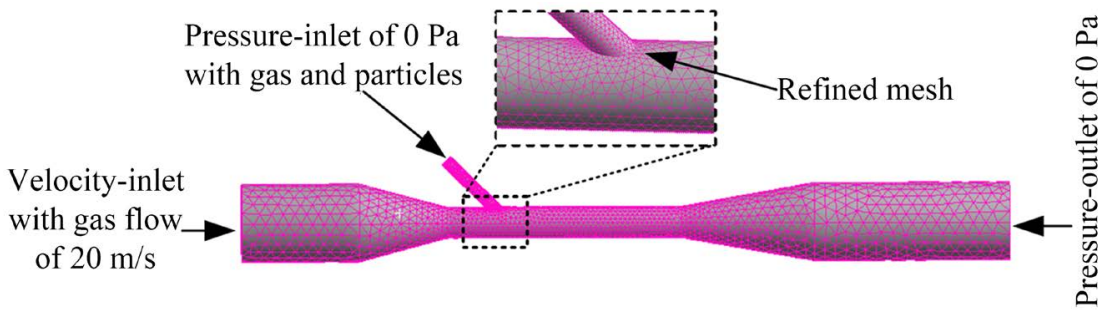

Figure 3. Simulation model mesh and initial condition. 


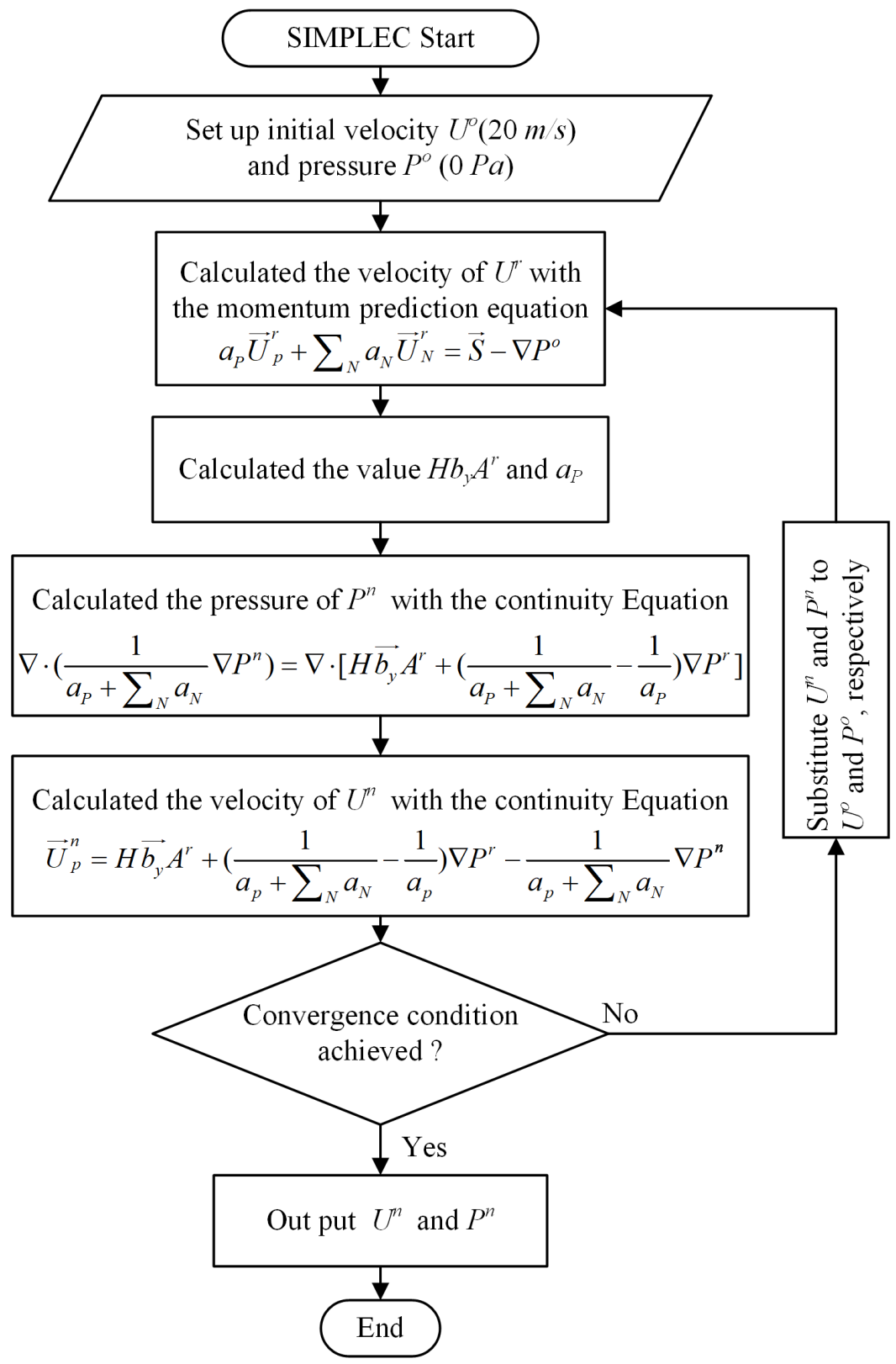

Figure 4. The solving process of velocity and pressure by SIMPLEC algorithm.

$$
\begin{gathered}
\nabla \cdot\left(\frac{1}{a_{P}+\sum_{N} a_{N}} \nabla P^{n}\right)=\nabla \cdot\left[H \overrightarrow{b_{y}} A^{r}+\left(\frac{1}{a_{P}+\sum_{N} a_{N}}-\frac{1}{a_{P}}\right) \nabla P^{r}\right] \\
\overrightarrow{U_{p}^{n}}=H \overrightarrow{b_{y}} A^{r}+\left(\frac{1}{a_{P}+\sum_{N} a_{N}}-\frac{1}{a_{P}}\right) \nabla P^{r}-\frac{1}{a_{P}+\sum_{N} a_{N}} \nabla P^{n}
\end{gathered}
$$

The variation parameters include $k_{1}, k_{2}, \alpha, \beta$. A single variable method was used. $k_{1}$ and $k_{2}$ ranging from 0.4 to 0.9 with a step of $0.1, \alpha$ ranging from $5^{\circ}$ and $30^{\circ}$ with a step of $5^{\circ}, \beta$ ranging from $4^{\circ}$ to $14^{\circ}$ with a step of $2^{\circ}$. Details see in Table 2. After finish the first part of our simulation, a verification mode is used to assess its performance. 
Table 2. The parameters and simulation arrangement.

\begin{tabular}{|c|c|c|c|c|}
\hline No. & $k_{1}$ & $k_{2}$ & $\alpha\left(^{\circ}\right)$ & $\beta\left(^{\circ}\right)$ \\
\hline 1 & 0.4 & 0.4 & 15 & 10 \\
\hline 2 & 0.4 & 0.5 & 15 & 10 \\
\hline 3 & 0.4 & 0.6 & 15 & 10 \\
\hline 4 & 0.4 & 0.7 & 15 & 10 \\
\hline 5 & 0.4 & 0.8 & 15 & 10 \\
\hline 6 & 0.4 & 0.9 & 15 & 10 \\
\hline 7 & 0.5 & 0.4 & 15 & 10 \\
\hline 8 & 0.6 & 0.4 & 15 & 10 \\
\hline 9 & 0.7 & 0.4 & 15 & 10 \\
\hline 10 & 0.8 & 0.4 & 15 & 10 \\
\hline 11 & 0.9 & 0.4 & 15 & 10 \\
\hline 12 & 0.4 & 0.5 & 5 & 4 \\
\hline 13 & 0.4 & 0.5 & 10 & 4 \\
\hline 14 & 0.4 & 0.5 & 15 & 4 \\
\hline 15 & 0.4 & 0.5 & 20 & 4 \\
\hline 16 & 0.4 & 0.5 & 25 & 4 \\
\hline 17 & 0.4 & 0.5 & 30 & 4 \\
\hline 18 & 0.4 & 0.5 & 5 & 6 \\
\hline 19 & 0.4 & 0.5 & 5 & 8 \\
\hline 20 & 0.4 & 0.5 & 5 & 10 \\
\hline 21 & 0.4 & 0.5 & 5 & 12 \\
\hline 22 & 0.4 & 0.5 & 5 & 14 \\
\hline
\end{tabular}

\section{Results and Discussion}

Figure 5 reported the pressure distribution along the flow direction. It can be seen that static pressure initially falls as medium flow in the tubes, when arrives at the end of throat, the static pressure reaches its valley -1090 Pa. It is interesting to observe that the minimum static pressure achieves after medium pass through the throat. This could be explained by the absorption of extra air in the suction port, which caused oscillation. Figure 5(b) reported the static pressure distribution in the suction port projected to the axis of main tube. The pressure is overall in a stable state, ranging from -840 to $-949 \mathrm{~Pa}$. The pressure in the intersection part of suction pipe and throat pipe was significantly lower than the other parts of suction port. Hence, absorbing materials is become possible.

As mentioned above, the airflow velocity was closely related to the ratio of throat diameter to inlet diameter $\left(k_{1}\right)$, which controlled the cross-sectional area, under steady flow conditions. And it was confirmed again in Figure 6(a), where $k_{1}$ increased from 0.4 to 0.9 and the maximum airflow velocity of Venturi tube 


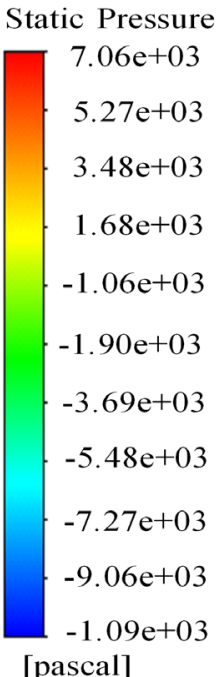

[pascal]

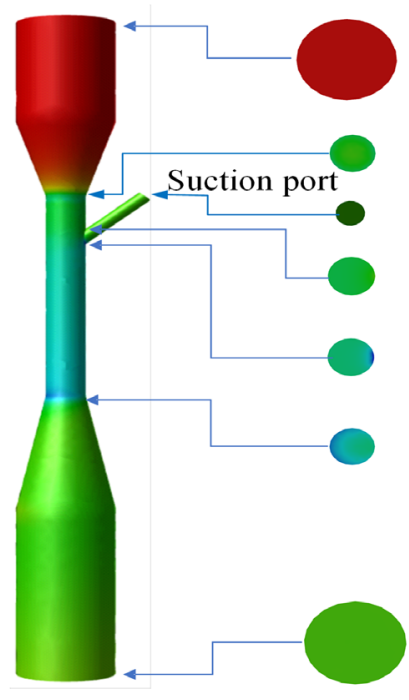

(a)

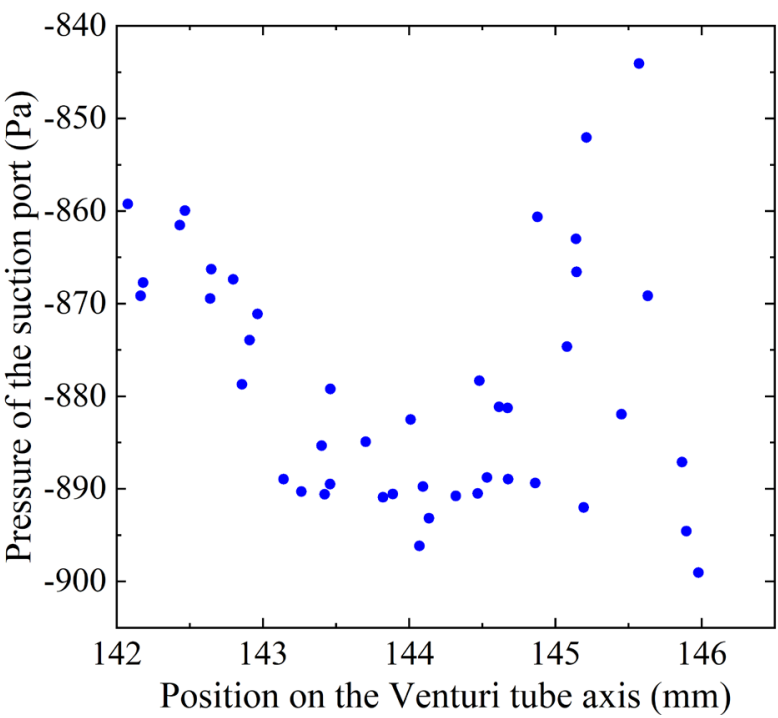

(b)

Figure 5. The pressure distribution of Venturi tube with $k_{1}=k_{2}=0.4, \alpha=15^{\circ}, \beta=10^{\circ}$ : (a) The total and local pressure nephogram, (b) Pressure distribution in the suction port.

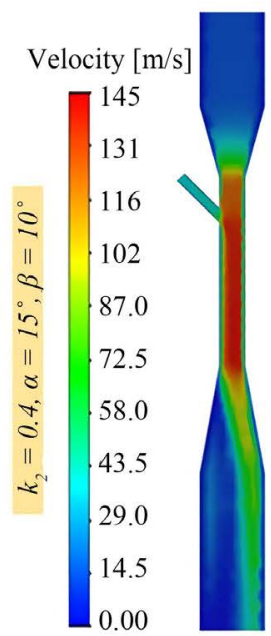

(a) $k_{l}=0.4$

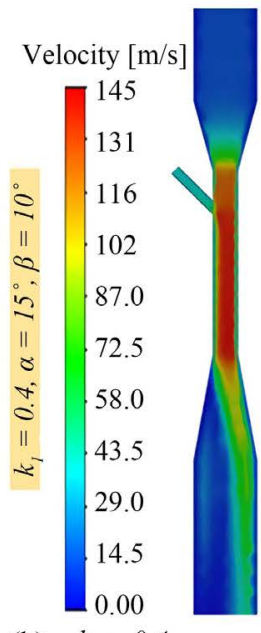

(b) $k_{2}=0.4$

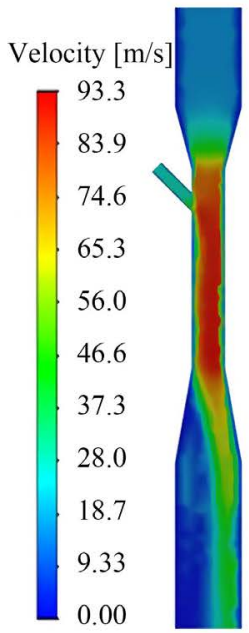

$k_{1}=0.5$

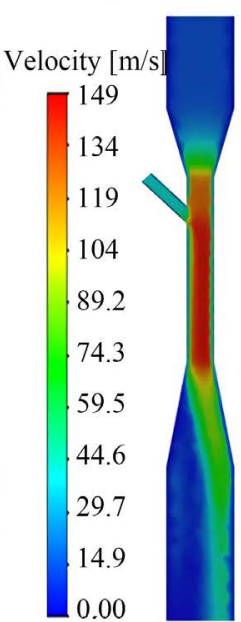

$k_{2}=0.5$

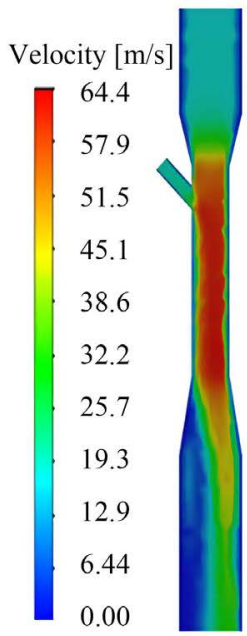

$k_{1}=0.6$

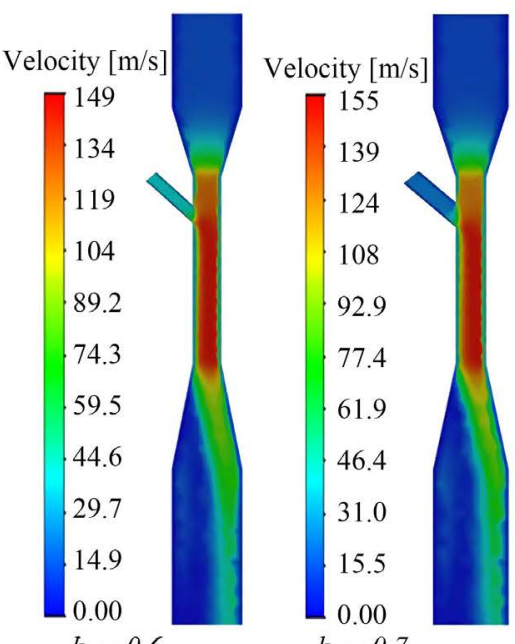

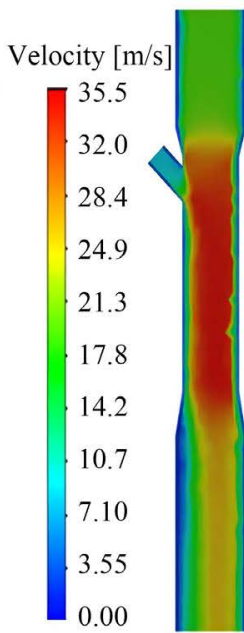

$k_{1}=0.8$

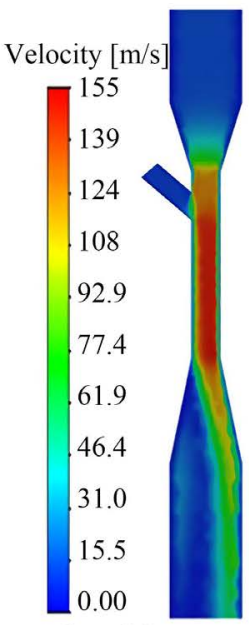

$k_{2}=0.8$

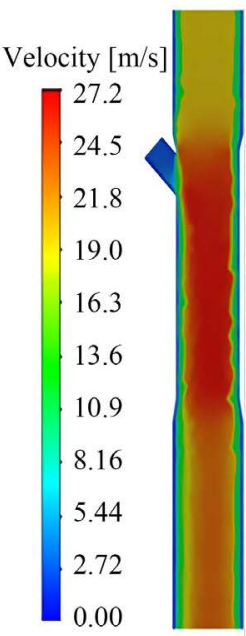

$k_{1}=0.9$

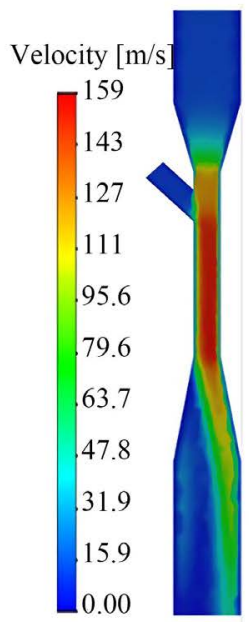

$k_{2}=0.9$ 


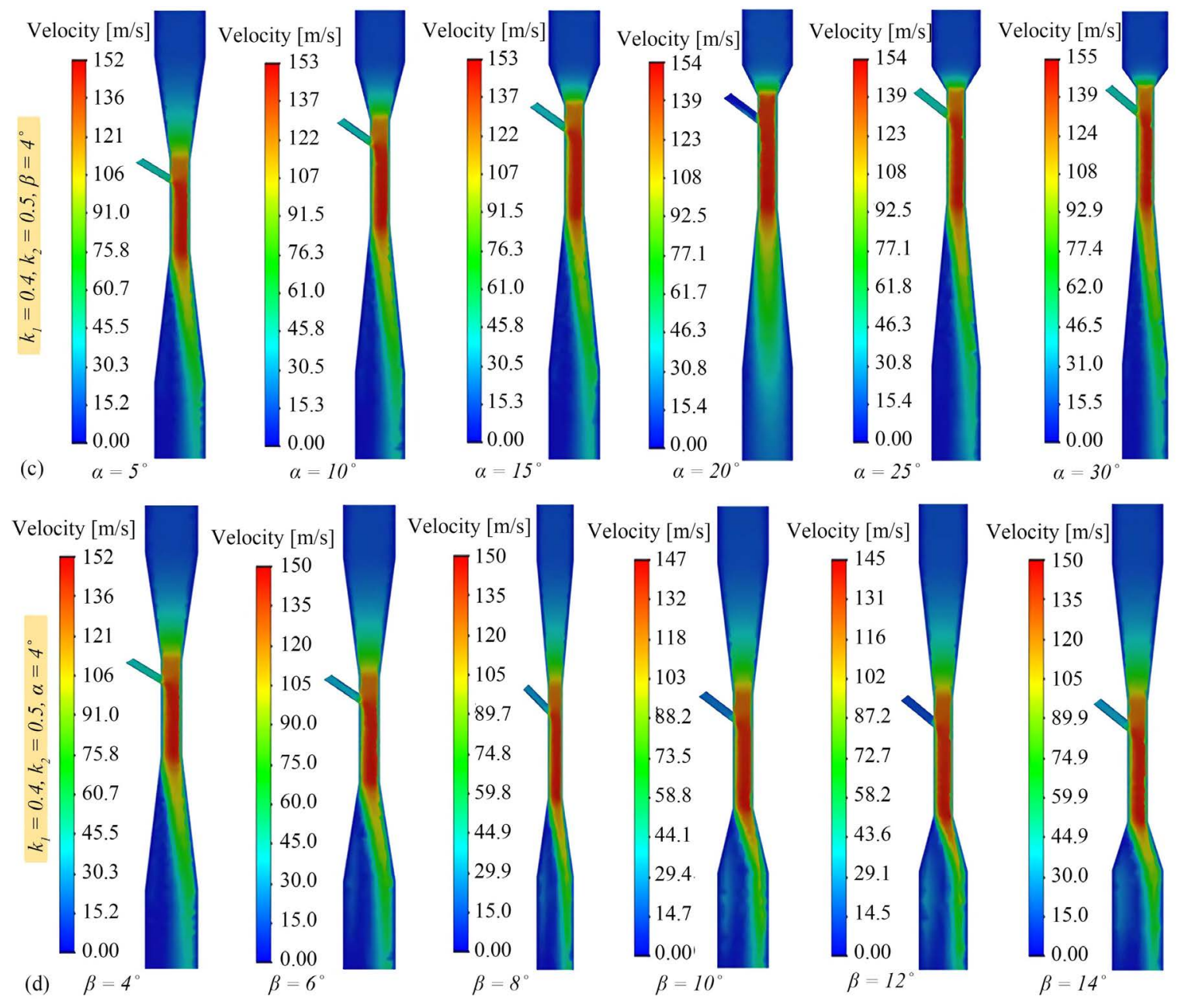

Figure 6. The distribution of velocity contour with different parameters: (a) $k_{1}$ goes from 0.4 to 0.9 with a step of 0.1 ; (b) $k_{2}$ goes from 0.4 with a step of 0.1 ; (c) $\alpha$ goes from $5^{\circ}$ to $30^{\circ}$; (d) $\beta$ goes from $4^{\circ}$ to $14^{\circ}$.

decreased from $145 \mathrm{~m} / \mathrm{s}$ to $27.2 \mathrm{~m} / \mathrm{s}$. But other variables have little effect on maximum velocity compare with $k_{1}$. Moreover, the maximum velocity increases as $k_{2}$ increases (See Figure 6(b)), which is opposite to the trend in Figure 6(a). When $k_{2}$ increases, the diameter of suction pipe will also increase. Then the fluid drag force that courses by the suction pipe will decrease and leading to the increasing of maximum velocity in Venturi tube. An interesting fact that the velocity distribution of diffuser section is symmetrical when the convergent angle is $20^{\circ}$ (See Figure $6(\mathrm{c})$ ), whereas the high velocity of the others spread over the position that is opposite to the side of suction port when parameters change. No obvious velocity change can be found with the change of diffuser angle from $4^{\circ}$ to $14^{\circ}$ (See Figure 6(d)). Hence, a conclusion can be drawn that the value of diffuser angle has the least effect on the airflow velocity of Venturi tube among the four factors in this study.

\subsection{Diameter Ratio Comparison}

Axial airflow velocity in the intersection of suction pipe and throat pipe versus $k_{1}$ 
was reported in Figure 7. $k_{1}$ ranges from 0.4 to 0.9 with a step of 0.1 . It can be seen that the airflow velocity is increasing as much as $k_{1}$ decreasing. This can be explained that when the inlet velocity and flow rate kept constant, the increase of $k_{1}$ will lead to an increase in the throat diameter, which in turn causes a decrease on airflow velocity. It is noteworthy that the airflow velocity is lower than $30 \mathrm{~m} / \mathrm{s}$ and decreases with increasing distance from $\mathrm{A}$ when $k_{1}=0.7$, which is an idealess outcome. When $k_{1}$ ranges from 0.7 to 0.9 , the airflow velocity is at a low value without any changing. But when $k_{1}=0.4$, the airflow velocity is significantly improved, which can reach a highest value of $84.5 \mathrm{~m} / \mathrm{s}$. Hence, $k_{1}=0.4$ is the best choice for the Venturi tube that used for mixed abrasive transportation in current study.

As indicated by Figure 8, axial airflow velocity in the intersection of suction pipe and throat pipe as function of $d_{i}$ has been studied. The flow velocity is increasing as much as the distance from point A increasing. Moreover, we can observe that airflow velocity is almost a constant and always at a low value when the distance from A more than $2.5 \mathrm{~mm}$ and $k_{2}$ of 0.4 and 0.9 have been selected. However, when the other distance from A less than $2.5 \mathrm{~mm}$, the airflow velocity increases very quickly. A $k_{2}$ of 0.5 or 0.6 has a significant impact on the airflow velocity, which results in a quickly increasing of airflow velocity. Moreover, a highest airflow velocity of $76.3 \mathrm{~m} / \mathrm{s}$ appears when a $k_{2}$ equal to 0.5 . Based on the above analysis, $k_{2}=0.5$ is the best choice, which can create largest negative pressure and improve the efficiency of the proposed device.

Except for airflow velocity, the pressure distribution is also an important index to evaluate the performance of such device. The minimum pressure on CD line varies with $k_{1}$ and $k_{2}$, which ranges from 0.4 to 0.9 , is illustrated in Figure 9. When $x=1$, then $k_{1}$ is 0.4 to 0.9 with a step of 0.1 and $k_{2}$ is 0.4 . While $\mathrm{x}=2$, then $k_{1}$ is 0.4 , and $k_{2}$ is 0.4 to 0.9 with a step of 0.1 . The minimum pressure is gradually

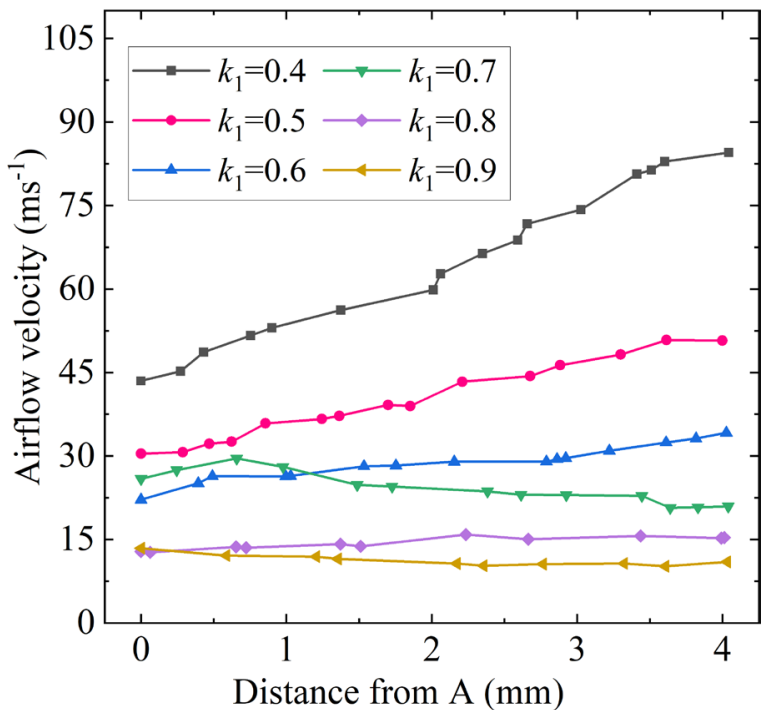

Figure 7. The velocity distribution on $\mathrm{AB}$ line with $k_{2}=0.4, \alpha=15^{\circ}, \beta=8^{\circ}$ and $k_{1}$ is 0.4 to 0.9 . 


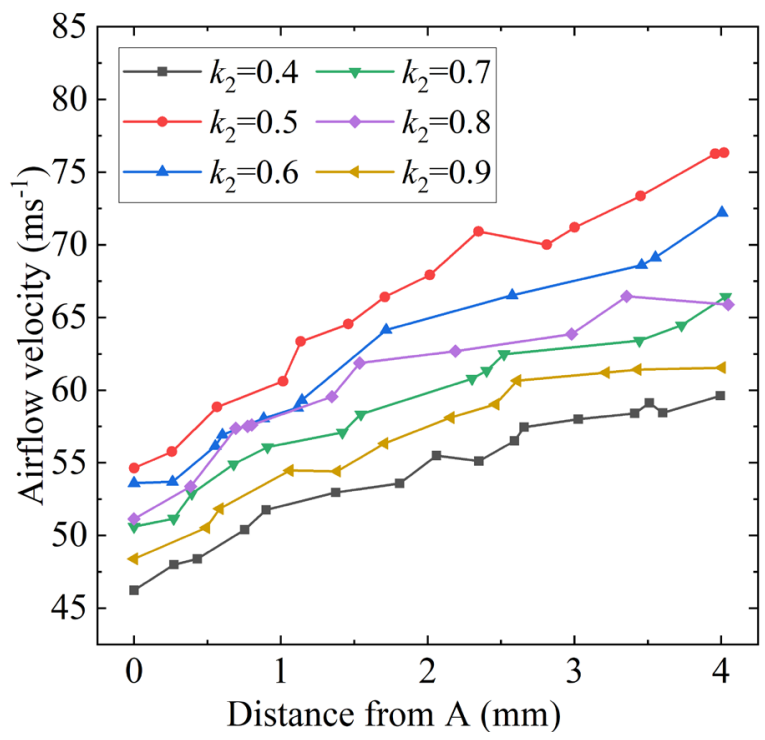

Figure 8. The velocity distribution on $\mathrm{AB}$ line with $k_{1}=0.4, \alpha=15^{\circ}, \beta=8^{\circ}$ and $k_{2}$ is 0.4 to 0.9 .

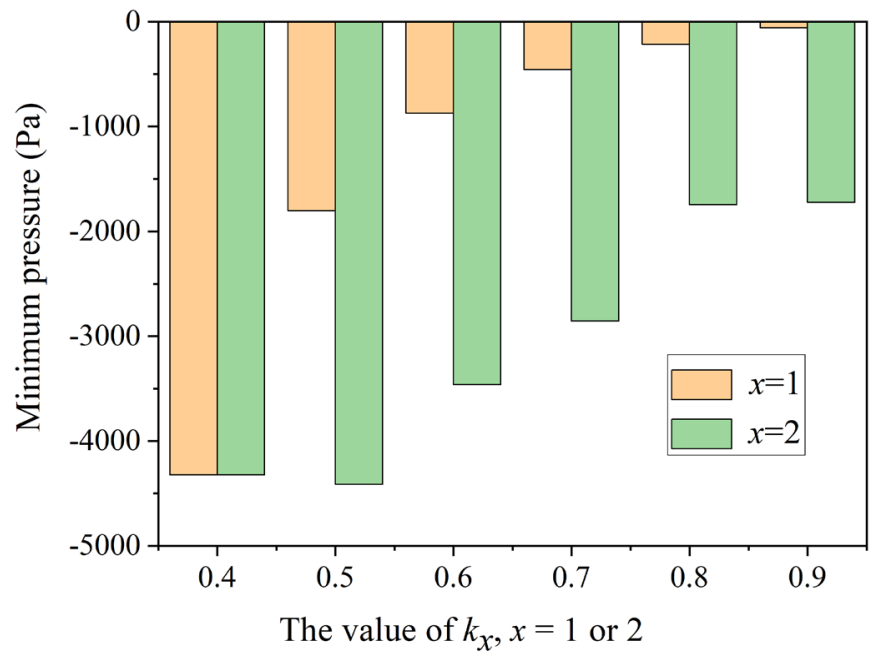

Figure 9. The variation of the minimum pressure on CD line with $k_{x}$, which $x$ is 1 or 2 .

increasing as $k_{1}$ increasing. It can be explained that the increase in $k_{1}$ will increase the diameter of the throat when $k_{2}$ remains unchanged, which in turn causes the flow velocity to decrease and the pressure to rise [23] [24]. But with the increasement of $k_{2}$, the minimum pressure decreases slightly and then increases. The change trend of minimum pressure is in agreement with the airflow velocity distribution trend of Figure 6 . At the $k_{2}$ of 0.5 , the minimum pressure reaches its lowest value $\sim-4414.09 \mathrm{~Pa}$. From here, we can observe that $k_{2}$ equal to 0.7 is a compromise. Furthermore, 0.4 and 0.5 are found to be the optimal selection of $k_{1}$ and $k_{2}$, respectively.

\subsection{Convergent and Diffuser Angle Comparison}

Furthermore, convergent angle $(\alpha)$ and diffuser angle $(\beta)$ also influence the per- 
formance of the proposed Venturi tube (See in Figure 6(a) and Figure 6(b)), which also need to take into consideration. Then the airflow velocity versus different $\alpha$ and $\beta$ have also been investigated (See Figure 10). The $\alpha$ is range from $5^{\circ}$ to $30^{\circ}$ with a step of $5^{\circ}$ and the $\beta$ is range from $4^{\circ}$ to $14^{\circ}$ with a step of $2^{\circ}$. Compared with other parameters, $\alpha$ and $\beta$ have a relatively small impact on the airflow velocity, which is similar to the results reported by Haifeng $\mathrm{Lu}$ et al. [25].

We can observe that the airflow velocities increase while the distances from point A increase (See Figure 10), which is similar to Figure 7 and Figure 8. Moreover, when $\alpha$ ranges from $5^{\circ}$ to $20^{\circ}$, the airflow velocity is increasing as much as $\beta$ increasing, while the opposite trend is found with $\alpha$ ranges from $20^{\circ}$ to $30^{\circ}$. The higher velocity is more benefit for particle transportation in the venturi tube [26]. Hence, $\alpha=20^{\circ}$ has been selected since it possesses the highest airflow velocity of $90.5 \mathrm{~m} / \mathrm{s}$ (See Figure 10(a)). The airflow velocity is significantly higher than the others when $\beta$ equal to $4^{\circ}$ (See Figure 10(b)). This is due to that the larger diffuser angle, the greater the energy loss, which causes by the boundary layer separation and eddy effects [27]. Even so, the larger diffuser angles also induce velocity sharply fluctuation that may reduce the kinetic energy. Since the higher velocity is more beneficial for mixed material conveying, the selection of diffuser angle should also take higher airflow velocity into account firstly. Finally, a convergent angle of $20^{\circ}$ and a diffuser angle of $4^{\circ}$ were selected.

\subsection{Verification of the Optimal Parameters}

In addition, comparative analysis between the optimal parameters and the others were then conducted for verifying the validity of the selection. As mentioned previously, $k_{1}=0.4, k_{2}=0.5, \alpha=20^{\circ}$ and $\beta=4^{\circ}$ are the optimal selections. To confirm the effectiveness of the optimization results, the selected optimal parameters were compared with the others in the previous analysis according to the airflow velocity and static pressure distribution (See in Figure 11 and Figure 12). We

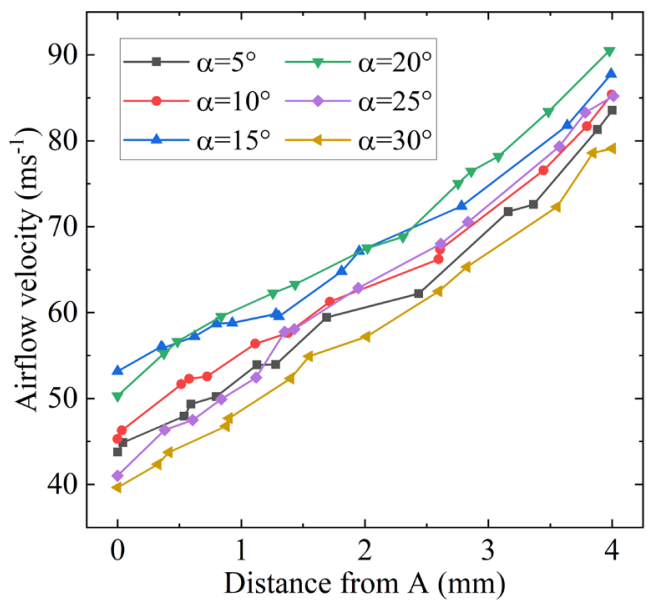

(a)

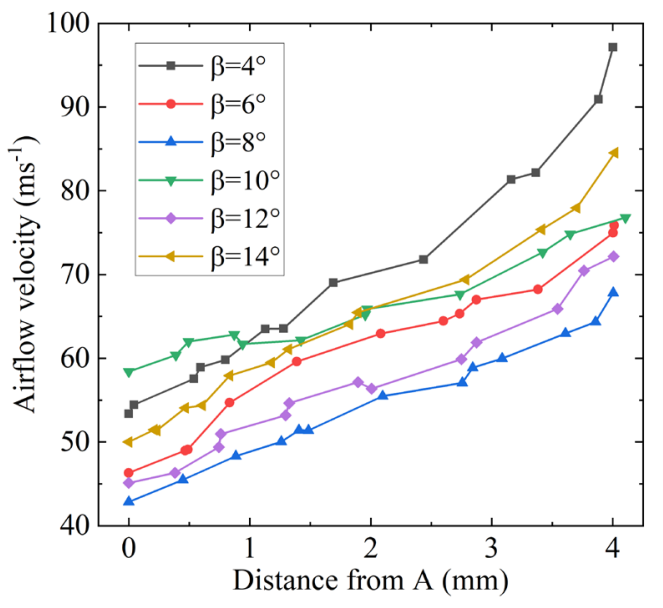

(b)

Figure 10. The velocity distribution on the $\mathrm{AB}$ line: (a) $\alpha$ changes from $5^{\circ}$ to $30^{\circ}$, (b) $\beta$ changes from $4^{\circ}$ to $14^{\circ}$. 


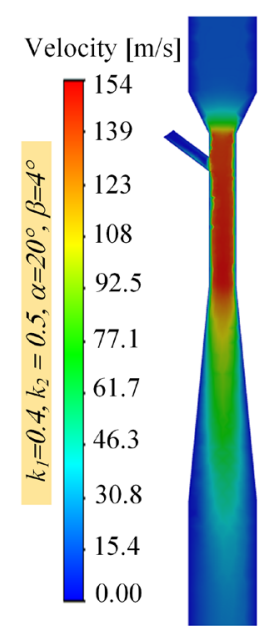

The optimal one
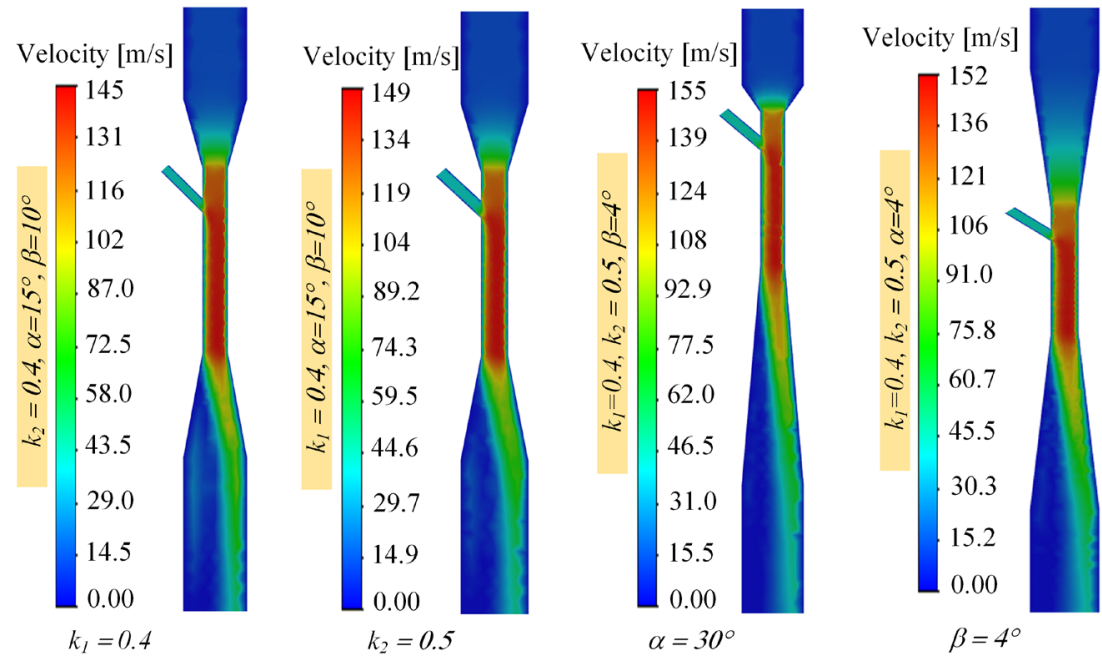

Figure 11. Velocity distribution comparison of the total optimal one and the single one.

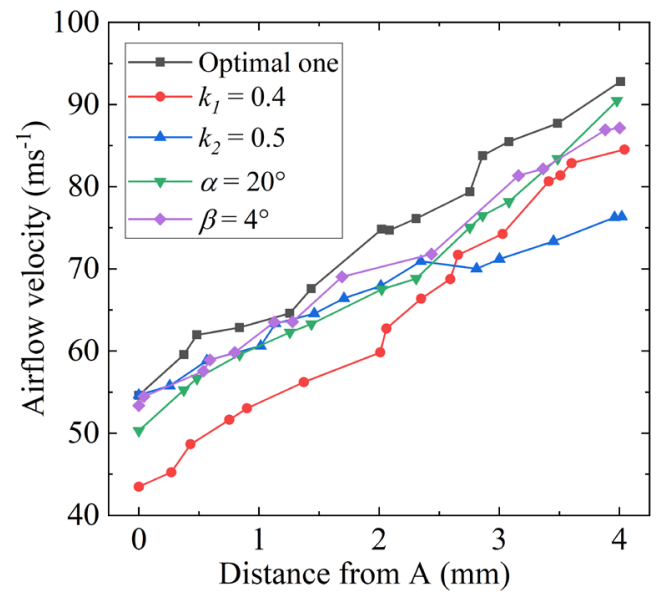

(a)

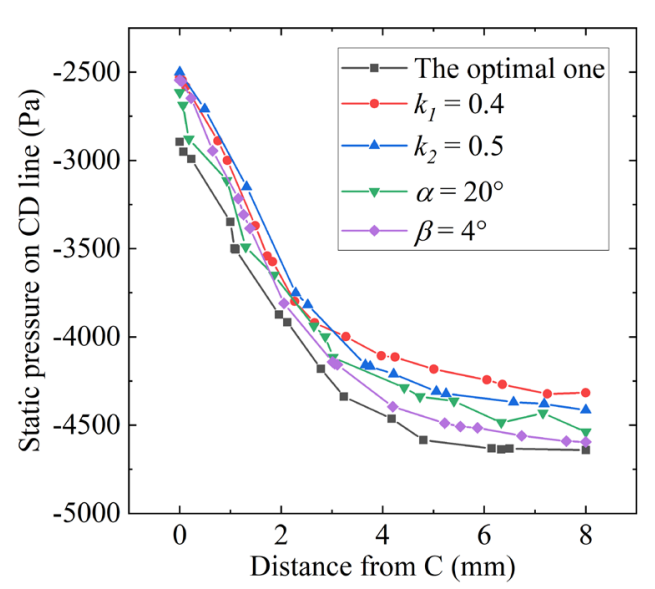

(b)

Figure 12. Velocity and pressure comparison of the total optimal one and single optimal one: (a) airflow velocity on $\mathrm{AB}$ line, (b) static pressure on $\mathrm{CD}$ line.

can find that the optimal one obtained the maximum airflow velocity of $154 \mathrm{~m} / \mathrm{s}$, which is only $1 \mathrm{~m} / \mathrm{s}$ lower that can be ignored comparing with the highest velocity. Moreover, the airflow velocity distribution curve on $A B$ line of the optimal one is higher than that of the others (See in Figure 12(a)). As a result, reasonable negative pressure appears on the $\mathrm{CD}$ line of the optimal one. In addition, the optimal one possesses the lowest negative pressure, which is in the range of -1550 $\mathrm{Pa}$ and $-1600 \mathrm{~Pa}$, in the suction port (See in Figure 13). It is consistent with the results shown in Figure 12. Moreover, to further verify the reliability of the optimal parameters, the acceleration coefficient $(\varepsilon)$ of the proposed Venturi tube is introduced, which is defined as shown in Equation (14).

$$
\varepsilon=\frac{U^{s}}{U^{o}} \times \frac{D_{2}}{D_{1}}
$$

where $U^{s}$ is the maximum velocity on $\mathrm{AB}$. 


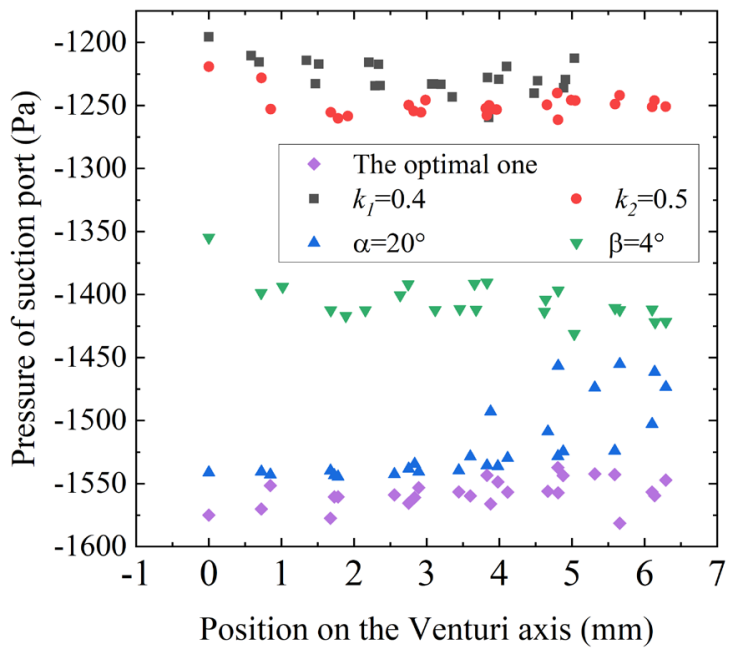

Figure 13. Comparison of pressure distribution on the suction port.

Table 3. Comparisons of acceleration coefficient.

\begin{tabular}{cc}
\hline Proposed structure & $\mathcal{\varepsilon}$ \\
\hline The optimal one in this study & 1.856 \\
Reference [16] & 0.542 \\
Reference [28] & 1.067 \\
Reference [29] & 1.057 \\
\hline
\end{tabular}

It can be seen from Equation (14) that the acceleration coefficient $(\varepsilon)$ represents the ratio of the product of the maximum velocity of the suction port and the diameter of the throat to the product of the inlet velocity and diameter. When the acceleration coefficient is larger, it means that the Venturi tube has a more significant speed-up effect, which is more conducive to the suction and transportation of mixed medium. Then the comparation of the optimal one and the others that proposed by references [16] [28] [29] were carried out, as shown in Table 3 .

Evidence can be found that the acceleration coefficient of the optimal one is 1.856, which is the greatest among the four. Furthermore, it's $73.4 \%$ higher than the next largest, which proposed in reference [28]. Obviously, the optimal one meets the requirements of higher speed. Hence, the selected parameters are approved to be credible for the Venturi tube of strengthen grinding equipment.

\section{Conclusions}

The geometric optimization of Venturi jet tubes for medium conveying in strengthen grinding equipment was performed by FEM. The following conclusions can be drawn based on the analysis.

When the diameter ratios of $k_{1}$ and $k_{2}$ are equal to 0.4 and 0.5 , a better acceleration effect and negative pressure effect achieve, which is more conducive to the transportation of medium during the strengthen grinding process. A convergence 
angle of $20^{\circ}$ and a diffuser angle of $4^{\circ}$ had selected for the optimal angle of the proposed Venturi tube in this study. The suction force mathematical model for medium conveying of different sizes particle by Venturi tube had been established, which could help the parameter design of Venturi tube.

A single-phase flow is used to simulate the flow field of the Venturi, and the rationality of the structure is evaluated by the flow velocity and pressure, which constitutes the limitations of this study. And the multiphase flow simulation and experiment need to be carried out in the future.

\section{Acknowledgements}

This research was supported by the Science and Technology Planning Project of Guangdong China (2019B020404) and the Science and Technology Planning Project of Guangzhou China (202102080225).

\section{Conflicts of Interest}

The authors declare no conflicts of interest regarding the publication of this paper.

\section{References}

[1] Long, X.P., Zhang, J.Q., Wang, J., Xu, M.S., Lyu, Q. and Ji, B. (2017) Experimental Investigation of the Global Cavitation Dynamic Behavior in a Venturi Tube with Special Emphasis on the Cavity Length Variation. International Journal of Multiphase Flow, 89, 290-298. https://doi.org/10.1016/j.ijmultiphaseflow.2016.11.004

[2] Brinkhorst, S., Lavante, E.V. and Wendt, G. (2015) Numerical Investigation of Cavitating Herschel Venturi-Tubes Applied to Liquid Flow Metering. Flow Measurement and Instrumentation, 43, 23-33. https://doi.org/10.1016/j.flowmeasinst.2015.03.004

[3] Bertoldi, D., Dallalb, C.C. and Barbosa, J.R. (2015) Experimental Investigation of TwoPhase Flashing Flows of a Binary Mixture of Infinite Relative Volatility in a Venturi Tube. Experimental Thermal and Fluid Science, 64, 152-163. https://doi.org/10.1016/j.expthermflusci.2015.02.011

[4] Kumar, R.K., Kumaran, P.S., Seetharamu, S., Kumar, S.A., Pramod, T. and Naveen, G.J. (2019) Investigation of Shot Peening Effect on Titanium Alloy Affecting Surface Residual Stress and Roughness for Aerospace Applications. Procedia Structural Integrity, 14, 134-141. https://doi.org/10.1016/j.prostr.2019.05.018

[5] Zhang, Z., Lin, M., Seng, D.H.L., Teo, S.L., Wei, F.X., Tan, H.R., Cheong, A.K.H., Lim, S.H., Wang, S.J. and Pan, J.S. (2020) Fatigue Life Enhancement in Alpha/Beta Ti6al-4v after Shot Peening: An EBSD and Tem Crystallographic Orientation Mapping Study of Surface Layer. Materialia, 12, Article ID: 100813. https://doi.org/10.1016/j.mtla.2020.100813

[6] Urazmetov, O., Cadet, M., Teutsch, R. and Antonyuk, S. (2021) Investigation of the Flow Phenomena in High-Pressure Water Jet Nozzles. Chemical Engineering Research and Design, 165, 320-332. https://doi.org/10.1016/j.cherd.2020.10.030

[7] Kanesan, D., Mohyaldinn, M.E., Ismail, N.I., Chandran, D. and Liang, C.J. (2019) An Experimental Study on the Erosion of Stainless Steel Wire Mesh Sand Screen Using Sand Blasting Technique. Journal of Natural Gas Science and Engineering, 65, 267 
274. https://doi.org/10.1016/j.jngse.2019.03.017

[8] Liu, X.C., Huang, J.F., Liang, Z.W., Huang, W.F., Zhu, R., Gao, W.L. and Xiao, J.R. (2020) Preparation and Properties of the Composite Enhancement Layer of Bearing Ring. World Journal of Mechanics, 10, 139-153. https://doi.org/10.4236/wjm.2020.1010010

[9] Liang, Z.W., Xie, B.H., Liao, S.P. and Zhou, J.H. (2015) Concentration Degree Prediction of AWJ Grinding Effectiveness Based on Turbulence Characteristics and the Improved ANFIS. The International Journal of Advanced Manufacturing Technology, 80, 887-905. https://doi.org/10.1007/s00170-015-7027-0

[10] Liu, X.C., Liang, Z.W., Wen, G.L. and Yuan, X.F. (2019) Waterjet Machining and Research Developments: A Review. The International of Advanced Manufacturing Technology, 102, 1257-1335. https://doi.org/10.1007/s00170-018-3094-3

[11] Wu, H.T., Xu, Y., Wang, J.H., Zhang, T. and Wang, H.X. (2019) Study on the Similarity of Wet Gas Pressure Drop in Long-Throat Venturi. Flow Measurement and Instrumentation, 68, Article ID: 101580.

https://doi.org/10.1016/j.flowmeasinst.2019.101580

[12] Kurimoto, R., Nakazawa, K., Minagawa, H. and Yasuda, T. (2017) Prediction Models of Void Fraction and Pressure Drop for Gas-Liquid Slug Flow in Microchannels. Experimental Thermal and Fluid Science, 88, 124-133. https://doi.org/10.1016/j.expthermflusci.2017.05.014

[13] Wu, H.T., Xu, Y., Xiong, X.Q., Mamat, E., Wang, J.H. and Zhang, T. (2020) Prediction of Pressure Drop in Venturi Based on Drift-Flux Model and Boundary Layer Theory. Flow Measurement and Instrumentation, 71, Article ID: 101673. https://doi.org/10.1016/j.flowmeasinst.2019.101673

[14] Liu, K., Guo, X.L., Peng, G. and Xin, H.F. (2017) Design Optimization of a Venturi Tube Geometry in Dense-Phase Pneumatic Conveying of Pulverized Coal for EntrainedFlow Gasification. Chemical Engineering Research \& Design: Transactions of the Institution of Chemical Engineers, 120, 208-217. https://doi.org/10.1016/j.cherd.2017.02.020

[15] Li, M., Bussonni`ere, A., Bronson, M., Xu, Z. and Liu, Q.X. (2019) Study of Venturi Tube Geometry on the Hydrodynamic Cavitation for the Generation of Microbubbles. Minerals Engineering, 132, 268-274. https://doi.org/10.1016/j.mineng.2018.11.001

[16] Ji, D., Zhang, M.L., Xu, T.G., Wang, K.Y., Li, P.C. and Ju, F. (2015) Experimental and Numerical Studies of the Jet Tube Based on Venturi Effect. Vacuum, 111, 25-31. https://doi.org/10.1016/j.vacuum.2014.09.010

[17] Haider, A. and Levenspiel, O. (1989) Drag Coefficient and Terminal Velocity of Spherical and Nonspherical Particles. Powder Technology, 58, 63-70.

https://doi.org/10.1016/0032-5910(89)80008-7

[18] Soyama, H. (2021) Luminescence Intensity of Vortex Cavitation in a Venturi Tube Changing with Cavitation Number. Ultrasonics Sonochemistry, 71, Article ID: 105389. https://doi.org/10.1016/j.ultsonch.2020.105389

[19] Song, Y.C., Shen, Y., Qian, Y.L., Yin, J.L. and Wang, D.Z. (2021) Experiment and Modeling of Liquid-Phase Flow in a Venturi Tube Using Stereoscopic PIV. Nuclear Engineering and Technology, 53, 79-92. https://doi.org/10.1016/j.net.2020.06.027

[20] Wu, H.T., Xu, Y., Wang, J.H., Yang, Y.G., Li, T., Zhang, T., Li, J.L. and Hao, C.J. (2021) Gas-Liquid Two-Phase Flowrate Measurement in Pseudo-Slug Flow with Venturi. Flow Measurement and Instrumentation, 78, Article ID: 101887.

https://doi.org/10.1016/j.flowmeasinst.2021.101887 
[21] Serra, N. and Semiao, V. (2021) ESIMPLE, a New Pressure-Velocity Coupling Algorithm for Built-Environment CFD Simulations. Building Environment, 204, Article ID: 108170. https://doi.org/10.1016/j.buildenv.2021.108170

[22] Aguerre, H., Venier, C.M., Pairetti, C.I., Damián, S.M. and Nigro, N.M. (2020) A SIMPLE-Based Algorithm with Enhanced Velocity Corrections: The COMPLEX Method. Computers \& Fluids, 198, Article ID: 104396.

https://doi.org/10.1016/j.compfluid.2019.104396

[23] Mishra, C. and Peles, Y. (2006) An Experimental Investigation of Hydrodynamic Cavitation in Micro-Venturis. Physics of Fluids, 18, Article ID: 103603. https://doi.org/10.1063/1.2360996

[24] Sharifi, A. and Mohebbi, A. (2014) A Combined CFD Modeling with Population Balance Equation to Predict Pressure Drop in Venturi Scrubbers. Research on Chemical Intermediates, 40, 1021-1042. https://doi.org/10.1007/s11164-013-1018-2

[25] Lu, H.F., Guo, X.L., Li, P., Liu, K. and Gong, X. (2017) Design Optimization of a Venturi Tube Geometry in Dense-Phase Pneumatic Conveying of Pulverized Coal for Entrained-Flow Gasification. Chemical Engineering Research and Design, 120, 208-217. https://doi.org/10.1016/j.cherd.2017.02.020

[26] Novo, P.G., Kyozuka, Y. and Villamayor, M.J.G. (2019) Evaluation of Turbulence-Related High-Frequency Tidal Current Velocity Fluctuation. Renewable Energy, 139, 313 325. https://doi.org/10.1016/j.renene.2019.02.035

[27] Liu, K., Lu, H.F., Guo, X.L., Sun, X.L., Tao, S.L. and Gong, X. (2014) Experimental Study on Flow Characteristics and Pressure Drop of Gas-Coal Mixture through Venturi. Powder Technology, 268, 401-411. https://doi.org/10.1016/j.powtec.2014.07.019

[28] Fang, L., Li, W., Li, Q. and Wang, Z.B. (2020) Numerical Investigation of the Cavity Shedding Mechanism in a Venturi Reactor. International Journal of Heat and Mass Transfer, 156, Article ID: 119835. https://doi.org/10.1016/j.ijheatmasstransfer.2020.119835

[29] Ahmed, S., Mohsin, H., Qureshi, K., Shah, A., Siddique, W., Waheed, K., Irfan, N., Ahmad, M. and Farooq, A. (2018) Investigation of Dust Particle Removal Efficiency of Self-Priming Venturi Scrubber Using Computational Fluid Dynamics. Nuclear Engineering and Technology, 50, 665-672. https://doi.org/10.1016/j.net.2018.01.016 